\title{
Orin Shanks
}

United States Environmental Protection Agency Cincinnati, United States

\section{Lindsay A. Peed}

United States Environmental Protection Agency Cincinnati, United States

\section{Catherine A. Kelty}

United States Environmental Protection Agency Cincinnati, United States

\section{Mano Sivaganesan}

United States Environmental Protection Agency

Cincinnati, United States

\section{Christopher T. Nietch}

United States Environmental Protection Agency

Cincinnati, United States 


\section{(c) (1) ()}

This publication is available in Open Access under the Attribution-ShareAlike 3.0 IGO (CC-BY-SA 3.0 IGO) license (http://creativecommons.org/licenses/by-sa/3.0/igo). By using the content of this publication, the users accept to be bound by the terms of use of the UNESCO Open Access Repositor (http://www.unesco.org/openaccess/terms-use-ccbysa-en).

Disclaimer:

Disclaimer:
The designations employed and the presentation of material throughout this publication do not imply the expression of any opinion whatsoever on the part of UNESCO The designations employed and the presentation of material throughout this publication do not imply the expression of any opinion whatsoever on the part of UNESCO expressed in this publication are those of the authors; they are not necessarily those of UNESCO and do not commit the Organization.

Citation:

Shanks, O.C., Peed, L.A., Kelty, C.A., Sivaganesan, M. and Nietch, C.T. (2019). Pollution Source-Targeted Water Safety Management: Characterization of Diffuse Human Fecal Pollution Sources with Land Use Informatino, Strategic Water Sampling, and Quantitative Real-Time PCR. In: J.B. Rose and B. Jiménez-Cisneros, (eds) Water and Sanitation for the 21st Century: Health and Microbiological Aspects of Excreta and Wastewater Management (Global Water Pathogen Project). (S. Petterson and G. Medema (eds) Part 5: Case Studies), Michigan State University, E. Lansing, MI, UNESCO. https://doi.org/10.14321/waterpathogens.83

Acknowledgements: K.R.L. Young, Project Design editor; Website Design: Agroknow (http://www.agroknow.com)

Last published: July 1, 2019 


\section{Summary}

\section{Highlights}

- This case study demonstrates the use of humanassociated microbial source tracking qPCR combined with low-order stream sampling, precipitation information, and a high-resolution land use-based geographic information system mapping to characterize trends in human fecal pollution
- Microbial source tracking qPCR testing reveals a significant correlation between septic system density and human fecal pollution concentrations in adjacent receiving waters.

- Successful microbial source tracking qPCR implementation requires a detailed problem definition formulated as a hypothesis that can be supported or refuted based on a carefully crafted experimental design.

- Combining microbial source tracking qPCR with routine $E$. coli monitoring improves water quality safety management.

\section{Graphical Abstract}

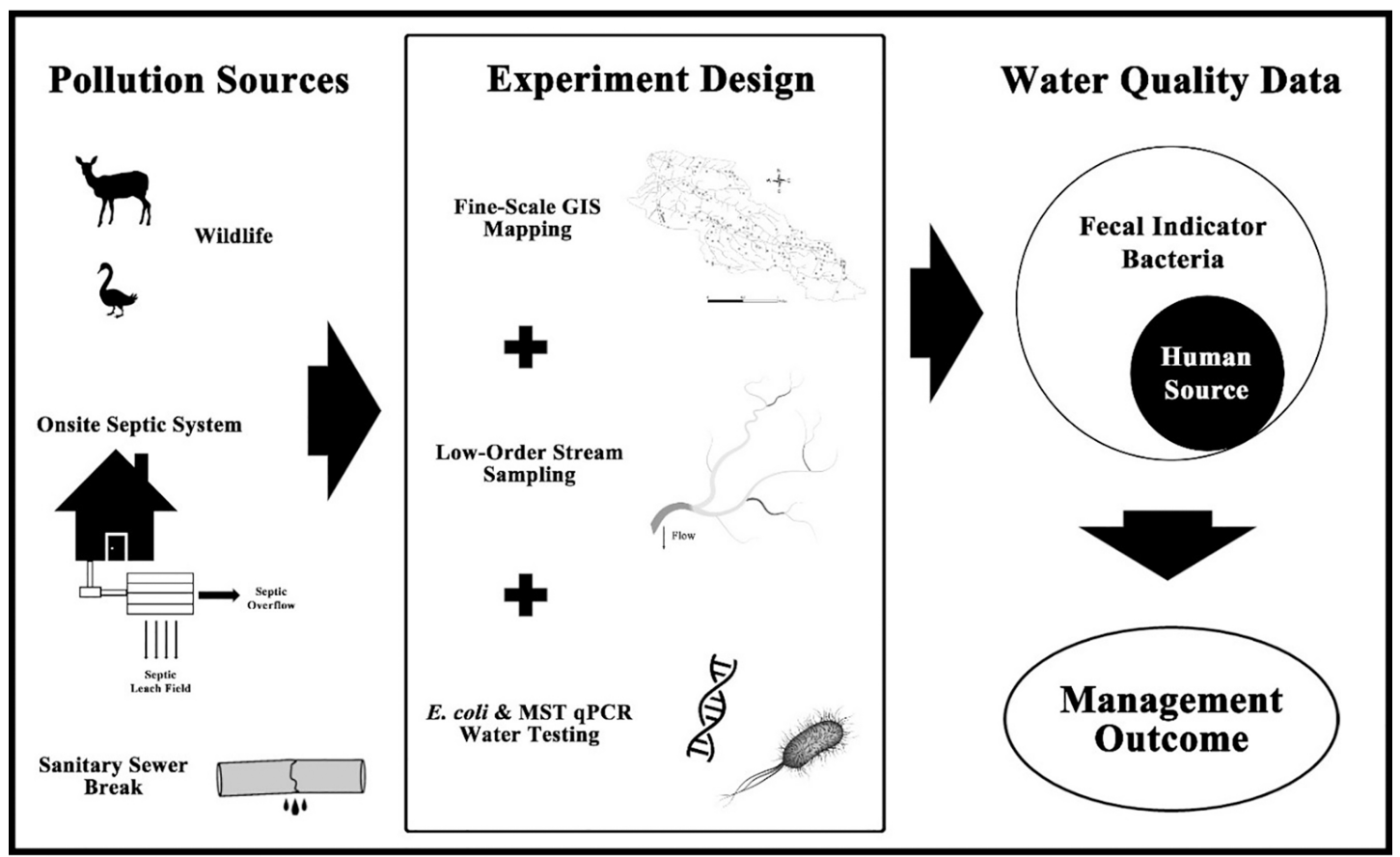

\section{Risk Management Objective}

The direct discharge of untreated human fecal pollution from diffuse sources can pose a serious health risk in recreational, drinking reservoir, irrigation, and aquaculture waters. Diffuse human fecal pollution can originate from a variety of sources such as leaky or damaged sewer lines, faulty septic systems, illicit waste disposal, and sanitary/combined sewer overflows. Evidence-based water safety management planning for diffuse fecal pollution typically relies on measurements of general fecal indicator bacteria (FIB), namely E. coli and enterococci providing an estimate of the concentration from all animal sources present in the area of interest.
Because many impaired waters are polluted by more than one animal source and FIB do cannot discriminate between pollution types, safety planning can be limited when relying solely on FIB measurements. Microbial source tracking (MST) can help discriminate between pollution sources to identify diffuse human fecal pollution. In this case study, human-associated MST methods are combined with low-order headwatershed sampling, precipitation information, and high-resolution geographic information land use data to identify diffuse sources of human fecal pollution. 


\section{Location and Setting}

This study was conducted in nine headwatersheds of the East Fork Watershed (EFW) situated in southeast Ohio, USA. Fecal pollution in the EFW headwatersheds is a chronic problem year round with FIB densities significantly increasing after wet weather events. Headwatersheds vary in size and land use intensity from densely forested to suburban with residential human waste management practices including septic systems and sanitary sewer lines.

\section{Description}

Due to limitations of FIB, it remains unclear whether fecal pollution in EFW headwaters originates from human waste management practices (sewer lines and/or septic systems) or other sources such as local wildlife. To characterize the presence of diffuse human fecal pollution, nine headwatersheds were sampled representing a gradient of human waste management practices (septic density of 0 to 89.4 counts $/ \mathrm{km} 2$ ) using local hydrology and highresolution geographic information land use mapping. MST measurements of human-associated genetic markers were then grouped by wet and dry weather conditions followed by correlation analysis to identify trends in diffuse human fecal pollution.

\section{Outcome and Recommendations}

The interdisciplinary strategy used in this study successfully identified septic system density during wet weather events as the likely source of diffuse human fecal pollution. In contrast, paired measurements of FIB did not correlate with septic density suggesting that chronic fecal pollution in EFW is not from human sources. Together these findings provide valuable pollution-source targeted information for water safety management planning to help improve water quality in the EFW.

\section{Introduction}

The discharge of untreated human fecal pollution from diffuse sources can pose a serious public health risk in recreational, drinking source, irrigation, and aquaculture waters. Diffuse human fecal pollution can originate from a variety of sources such as leaky or damaged sewer lines, faulty septic systems, and illicit waste disposal. Evidencebased water safety management planning for diffuse fecal pollution typically relies on measurements of general fecal indicator bacteria (FIB), namely E. coli and enterococci providing an estimate of the concentration from all sources present in an area of interest. Because many impaired waters are polluted by more than one source (e.g. sewage, agricultural waste, wildlife, etc.) and FIB cannot discriminate between pollution types, safety planning can be limited when relying solely on FIB measurements. Microbial source tracking (MST) technologies can help discriminate between pollution sources to identify diffuse human fecal pollution. In this case study, human-associated MST quantitative real-time PCR (qPCR) methods were combined with low-order stream sampling, precipitation information, and a high-resolution land use-based geographic information system (GIS) mapping to characterize trends in human fecal pollution diffuse sources on a watershed scale (Peed et al., 2011).

\section{Problem Formulation}

The East Fork Watershed (EFW) is situated in Southwestern, $\mathrm{OH}$ with mixed land uses including dense forest, row crop farming, and low to high density urban areas with many residential homes relying on septic systems for onsite waste management. The EFW is also home to wildlife including whitetail deer and several residential bird populations. Comparatively livestock densities are minimal. Routine water quality monitoring is conducted by local authorities using E. coli and when unsafe levels are observed in EFW receiving waters, it remains unclear whether fecal pollution originates from failing septic systems, leaky sewer lines, and/or local wildlife sources. Because $E$. coli is found in both human and wildlife fecal waste, the use of this general indicator provides limited information to a water quality manager seeking to mitigate fecal pollution in EFW. However, human-associated qPCR MST methods combined with a strategic watershed sampling plan and catchment land use information could be used to identify potential correlations between human fecal pollution and local waste management practices in EFW. 


\section{Experimental Design}

To identify potential correlations between the concentration of human fecal pollution in EFW receiving waters and the densities of septic systems and sewers, it is imperative to devise an experimental design tailored to address this question. In this case study, receiving waters from nine catchments were sampled over a two-year period. Catchment drainage boundaries were strategically chosen to contrast septic system and sewer line use (septic systems in \#/km2 and sewerlines in linear $\mathrm{ft} / \mathrm{km} 2$ ) as previously described (Peed et al., 2011). Because septic system and sewer line densities were negatively correlated $(r=-0.82 ; p=$ 0.007), the remainder of this case study focuses on septic system findings. Catchments ranged in size from 0.41 to 6.9 $\mathrm{km} 2$ and varied in septic system densities (0 to 89.4 count/ $\mathrm{km} 2$ ) representing a gradient of septic use across the study area providing an experimental framework to test the hypothesis that human fecal pollution concentrations are correlated with septic system use. In addition, receiving water sampling sites were strategically located at the furthest downstream drainage point intersecting a catchment boundary so that fecal pollution measurements were representative of upstream land use activities across the area of interest (see Figure 1 for example).

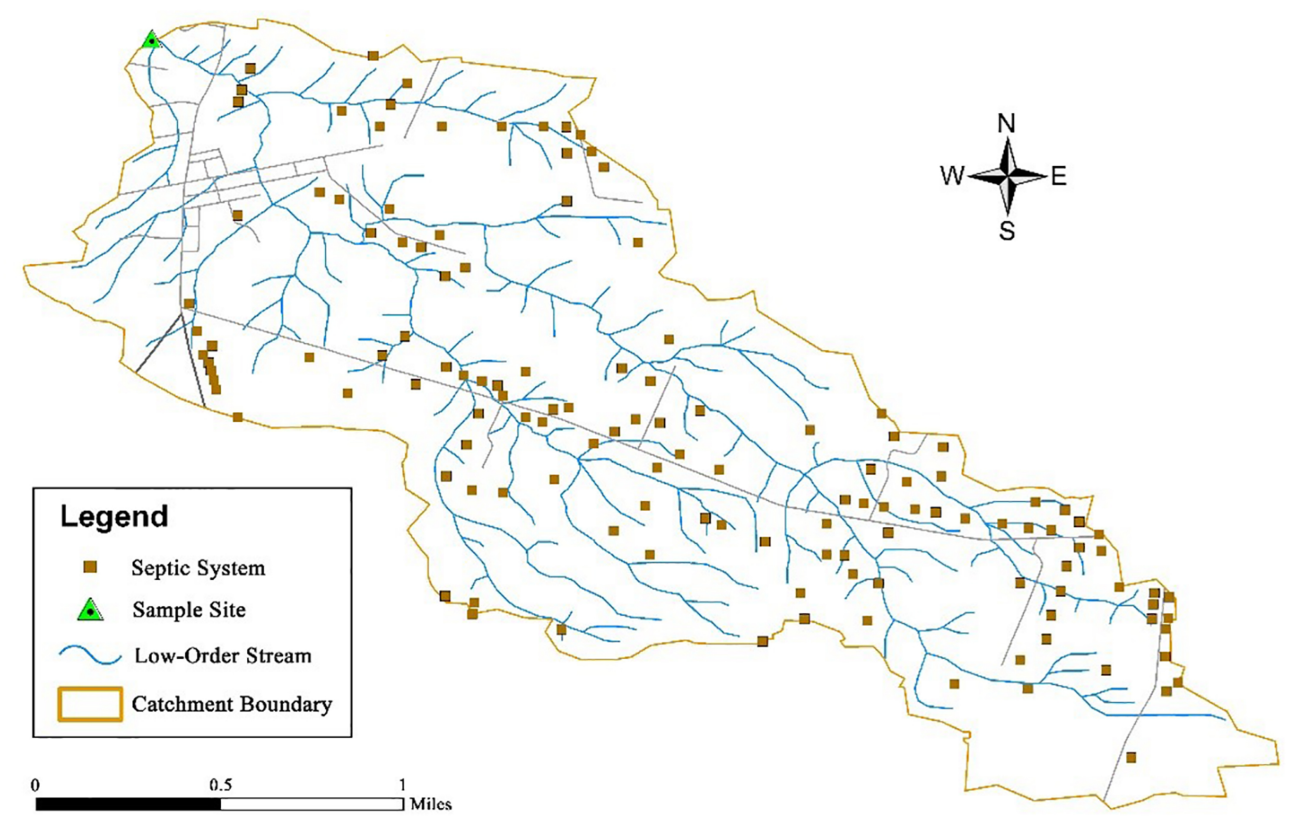

Figure 1: Example GIS-generated map showing topographically-defined catchment boundary, low-order stream drainage network, and septic system locations.

A total of 215 water samples were collected over the study period and subject to parallel testing for E. coli using the Colilert IDEEX procedure (IDEXX Laboratories, Inc.) and human fecal source identification with three humanassociated MST qPCR methods [Table 1; (Haugland et al., 2010)]. To explore the potential impact of wet weather on human fecal pollution in EFW streams, sampling events were categorized as dry or wet using continuous measures of local precipitation, in-stream water depth, and conductivity as previously described (Peed et al., 2011). Statistical analyses were then performed to identify any potential links between fecal pollution measurements (E. coli and human-associated qPCR) and septic system density during wet and dry conditions. 
Table 1. Summary of human-associated MST qPCR methods used in case study

\begin{tabular}{|c|c|c|c|}
\hline \multirow{2}{*}{ Assay } & \multirow{2}{*}{$\begin{array}{c}\text { Target } \\
\text { Organism }\end{array}$} & \multicolumn{2}{|r|}{ Primers and Probe } \\
\hline & & Name & Sequence $\left(5^{\prime}\right.$ to $\left.3^{\prime}\right)$ \\
\hline \multirow{3}{*}{ HF183 } & Bacteroides HF8 & HF183 & ATCATGAGTTCACATGTCCG \\
\hline & $\mathrm{CI}_{1}$ & BFDrev & CGTAGGAGTTTGGACCGTGT \\
\hline & & BFDrev & FAM-CTGAGAGGAAGGTCCCCCACATTGGA-TAMRA \\
\hline \multirow{3}{*}{ Bsteri } & Bacteroides & BfragF1 & CATTATTAAAGGATTCCGGTAAAG \\
\hline & fragilis & BFDrev & CGTAGGAGTTTGGACCGTGT \\
\hline & & BFDFam & FAM-CTGAGAGGAAGGTCCCCCACATTGGA-TAMRA \\
\hline \multirow[t]{3}{*}{ Btheta } & $\begin{array}{c}\text { Bacteroides } \\
\text { thetaiotaomicron }\end{array}$ & BsteriF1 & GGGATRATTATTAAAGAATTTCGGCTGT \\
\hline & & BFDrev & CGTAGGAGTTTGGACCGTGT \\
\hline & & BFDFam & FAM-CTGAGAGGAAGGTCCCCCACATTGGA-TAMRA \\
\hline
\end{tabular}

aThe HF8 cluster represents a group of human-associated 16S rRNA sequences (Bernhard and Field, 2000).

\section{Outcome and Recommendations}

Culture-based water quality testing for $E$. coli indicated that low-order EFW streams exceeded the U.S. Environmental Protection Agency recommended Ambient Water Quality Criteria of 235 MPN/100mL (USEPA, 1986) $33 \%$ to $77 \%$ of the time indicating that all catchments experienced unsafe fecal pollution levels over the study period. The experimental design successfully identified a significant positive correlation between septic system density during wet weather events and human fecal pollution concentrations for all three human-associated MST qPCR methods ( $r=+0.74$ to $+0.75 ; \mathrm{p} \leq 0.024)$. In contrast, paired measurements of $E$. coli did not correlate with septic density $(p>0.05)$, but they did significantly increase after rain events $(p<0.05)$ suggesting that fecal pollution in wet-weather flows in the EFW is not entirely from failing septic systems.

Together these findings demonstrate the benefit of using pollution-source targeted water quality testing compared to relying completely on an E. coli FIB approach. Results provide several new lines of evidence for local water quality safety managers. For instance, the presence of human fecal pollution was confirmed with three human- associated qPCR MST methods and was significantly correlated with catchment septic density, but only after wet weather events. This suggests that rainfall/runoff intrusion into existing septic systems is short-circuiting onsite treatment processes. This can be a problem for systems installed in poorly draining soils. In fact, it was determined from the local Health Department responsible for septic system oversight in the EFW that many of the active systems were of a variety that do not require a leach-field and instead rely on aeration for treatment (cavitation systems). This type of septic system directly discharges into the environment and is prone to failure without routine maintenance. Thus, the data from this study provides a manager with evidence that would support a need for septic system upgrades or a change to a centralized waste treatment approach. In addition, the absence of a correlation between septic density and $E$. coli implies that wildlife may also be a contributing factor to poor water quality and that future sanitary surveys may benefit from the inclusion of local bird and deer population counts. 


\section{Implications for Water Quality Management}

Identification of diffuse fecal pollution sources can be a significant component for water quality safety management, but currently represents a key challenge for many authorities, especially in mixed use watersheds with agricultural, wildlife, and residential areas. Advances in applied molecular biology now offer tools that can estimate the concentration of human and other animal fecal pollution sources in a water sample [for review see (Harwood et al., 2017)]. However, it is important to note that the successful identification of diffuse fecal pollution sources requires more than host-associated qPCR measurements. To establish a link between human fecal pollution concentrations and septic system density in this case study, it was necessary to construct an interpretation framework consisting of a detailed characterization of catchment and stream hydrology, rainfall, land use, and septic system location data. Stream sampling sites were selected based on multiple geodata sources integrated with high spatial resolution GIS mapping. Pairing precipitation data with in-stream flow and water quality measurements was essential for distinguishing wet-weather flow from baseflow sampling events. Unfortunately, these types of data may not be readily available making it more challenging to implement MST methods in some areas.

Another critical factor for the successful characterization of diffuse pollution in this study was a focus on low-order stream sampling. The more common practice of sampling larger water bodies (e.g. lakes and rivers), often to meet regulatory needs, makes it difficult to correlate nearby land use activities to water quality measurements because spatial heterogeneity of potential fecal pollution sources often increases with drainage area size, and, therefore, stream order. Low-order stream sampling coupled with GIS allows for field survey designs that more directly connect water quality conditions to specific land use practices. In addition, pollutants typically have shorter residence times in low-order streams helping to minimize die-off and dilution effects making it easier to recover and measure minute concentrations of host-associated qPCR genetic markers. In summary, case study findings clearly demonstrate the benefit of combining MST qPCR approaches with routine E. coli monitoring to improve water quality safety management.
Disclaimer: Information has been subjected to U.S. EPA peer and administrative review and has been approved for external publication. Any opinions expressed in this paper are those of the authors and do not necessarily reflect the official positions and policies of the U.S. EPA. Any mention of trade names or commercial products does not constitute endorsement or recommendation for use.

Acknowledgements: This case study was derived from a previous research project, the results of which are published in the following: Peed, L.A., Nietch, C.T., Kelty, C.A., Meckes, M., Mooney, T., Sivaganesan, M., and Shanks, O.C. (2011). Combining land use information and small stream sampling with PCR-based methods for better characterization of diffuse sources of human fecal pollution. Environmental Science \& Technology 45: 5652-5659. 


\section{References}

Bernhard, A.E. and Field, K.G. (2000). Identification of Nonpoint Sources of Fecal Pollution in Coastal Waters by Using Host-Specific 16S Ribosomal DNA Genetic Markers from Fecal Anaerobes. Applied and Environmental Microbiology. 66(4), pp. 1587-1594.

Harwood, V.J., Shanks, O.C., Korajkic, A., Verbyla, M., Ahmed, W. and Iriate, M. (2017). General and host-associated bacterial indicators of faecal pollution. Global Water Pathogen Project. Michigan State University. doi: doi.org/10.14321/waterpathogens.6.

Haugland, R.A., Varma, M., Kelty, C.A., Peed, L., Sivaganesan, M. and Shanks, O.C. (2010). Evaluation of genetic markers from the 16S rRNA gene V2 region for use in quantitative detection of selected Bacteroidales species and human fecal waste by real-time PCR. Systematic and Applied Microbiology. 33, pp. 348-357.

Peed, L.A., Nietch, C.T., Kelty, C.A., Meckes, M., Mooney, T., Sivaganesan, M. et al. (2011). Combining land use information and small stream sampling with PCR-based methods for better characterization of diffuse sources of human fecal pollution. Environmental Science and Technology. 45, pp. 5659-5662.

USEPA (1986). Ambient water quality criteria; availability. Federal Register. 51 (45), Federal Register. pp. 8012-8016. 